line treatment option for malignant biliary obstruction (MBO) is limited. We conducted a meta-analysis to compare the performance of EUS-BD and endoscopic retrograde cholangiopancreatography-guided biliary drainage (ERCP-BD) as primary palliation of MBO.

Methods We searched several databases for comparative studies evaluating EUS-BD vs. ERCP-BD in primary drainage of $\mathrm{MBO}$ up to 30 November 2018. The primary outcome was treatment success. Secondary outcomes included adverse events, stent patency, stent dysfunction, reinterventions, procedure duration, and overall survival. Fixed- and random-effects models were used to calculate the pooled estimates.

Results Seven studies involving 445 patients were qualified for the final analysis (IDDF2019-ABS-0023 Figure 1). There was no difference in technical success (risk ratio [RR] 0.99; 95\% confidence interval [95\% CI]: 0.86-1.13), clinical success (RR 1.02; 95\% CI: 0.94-1.12) and total adverse events (RR 0.82; 95\% CI: $0.49-1.37$ ) between the 2 procedures (IDDF2019ABS-0023 Figure 2, 3). EUS-BD was associated with lower rates of post-procedure pancreatitis (RR 0.17 ; 95\% CI: $0.05-$ 0.53 ), stent dysfunction (RR 0.62; 95\% CI: $0.40-0.97$ ), and tumor in/overgrowth (RR $0.21 ; 95 \%$ CI: $0.07-0.70)$, but higher rates of post-procedure bleeding (RR 8.82 ; 95\% CI: 1.08-72.21) and bile peritonitis (RR 4.84; 95\% CI: 1.0622.07). No differences were noted in reinterventions (RR 0.53 ; 95\% CI: $0.22-1.25$ ), procedure duration (weighted mean difference -2.11 ; 95\% CI: $-9.51-5.29$ ), stent patency (hazard ratio [HR] 0.71 ; 95\% CI: $0.45-1.11$ ), and overall survival (HR 1.12; 95\% CI: 0.80-1.58) (IDDF2019-ABS-0023 Figure 4, 5).

Conclusions EUS-BD showed similar efficacy, safety, stent patency, and overall survival when compared with ERCP-BD for primary palliation of $\mathrm{MBO}$, whereas exhibiting several clinical advantages (lower risk of post-procedure pancreatitis, stent dysfunction, and tumor in/overgrowth) and disadvantages (higher risk of post-procedure bleeding and bile peritonitis). Large-scale, well-organized, head-to-head studies are warranted.

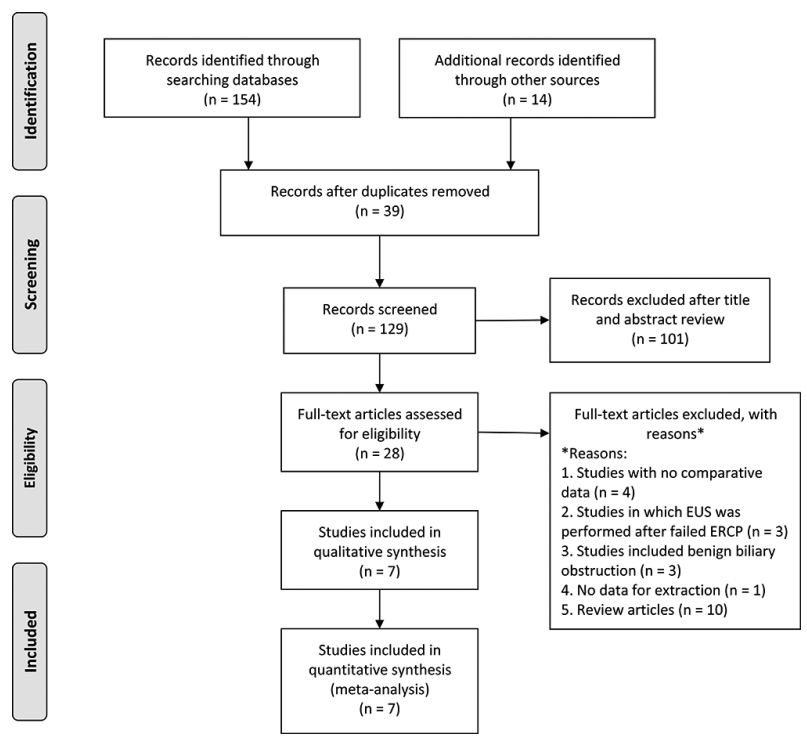

Abstract IDDF2019-ABS-0023 Figure 1
IDDF2019-ABS-0024 PREVALENCE OF HEPATITIS C AMONG TYPE 2 DIABETES MELLITUS PATIENTS IN A SINGLE CENTER TERTIARY HOSPITAL IN QUEZON CITY, PHILIPPINES

Millette Castro* . University of the East Ramon Magsaysay Memorial Medical Center, Philippines

10.1136/gutjnl-2019-IDDFabstracts.244

Introduction There is a growing body of literature between Hepatitis $C$ virus infection and T2DM on the framework of insulin resistance. However, there are gaps in the literature and data is still inconclusive. No local study on the prevalence of HCV among diabetics is available.

Objectives This is a pilot study that aims to determine the prevalence of $\mathrm{HCV}$ infection among diabetic patients seen at our institution.

Methodology This is a descriptive cross-sectional epidemiological study. Written consent was secured, a consecutive sampling of 318 known diabetics was done and data was collected using a checklist. A drop of whole blood was collected by the investigator by finger prick method and tested for $\mathrm{HCV}$ antibodies using RAPIDQuick screening test. Frequency and proportion and median and range were used to describe the clinical characteristics of the enrolled subjects. STATA 15.0 was used for data analysis.

Results 318 diabetic patients were tested for HCV. No HCV seropositivity was noted. The median age was 55 years and a half were female. $22 \%$ were obese. The following risk factors for HCV were reported: more than half had reported unprotected sex, shares nail cutters, toothbrush, and razors. 261 (82\%) had abnormal FBS and median HbA1c was 7.5\%. Similarly, $3 / 4$ had abnormal triglyceride levels. On average, more than half of the patients are insulin-requiring for 4 years have T2DM for four years.

Conclusions Despite a negative study, we would like to continue this study to determine the association between the two diseases and in the future can contribute to the local statistics of Hepatitis C.

\section{IDDF2019-ABS-0032 THE ANALYSIS OF THE ALLERGIC REACTION OF WOMEN IN THE THIRD TRIMESTER OF PREGNANCY INDUCED BY REDUCED GLUTATHIONE FOR INJECTION AND INOSINE INJECTION SUCCESSIVELY}

${ }^{1}$ Duan Xing Ping Duan*, 'Wang Yan Wang, ${ }^{2}$ Ren Yu Ren. 'Department of Pharmacy, Maternal and Child Health Care Hospital of Zigong, China; ${ }^{2}$ Department of Obstetrics and Gynecology, Maternal and Child Health Care Hospital of Zigong, China

\subsection{6/gutjnl-2019-IDDFabstracts.245}

Background The specific disease, intrahepatic cholestasis of pregnancy (ICP) is a common pregnancy-complication. However, there are no indications for ICP in the domestic licenced drugs for protecting the liver and reducing bile acid levels, which are often based on evidence-based medicine or evidence-based pharmacy to select drugs that have been used during pregnancy and have no teratogenic evidence.

Methods By analyzing the drug use (Reduced glutathione for injection, Inosine injection and Ursodeoxycholic acid tablets) and the allergic reactions in the clinical practice in a rare patient with hepatic disorders in third trimester of pregnancy, in order to remind clinicians that in the course of using hepatoprotective 